\title{
POLNISCHE EINWANDERER IN DER ÖSTERREICHISCHEN LITERATUR: EINIGE BEMERKUNGEN ÜBER DIE ÖSTERREICHISCH-POLNISCHEN LITERARISCHEN VERWANDTSCHAFTEN IM 21. JAHRHUNDERT
}

\begin{abstract}
Literaturgeschichte mit inter-/transkultureller Prägung zurück. Viele der österreichischen Autoren sind Grenzgänger zwischen den Kulturen, ihre interkulturelle Bindung verleiht ihnen eine „brückenschlagende“ Funktion: Die literarischen Texte von Autoren mit Migrationshintergrund bilden heute einen festen Bestandteil der österreichischen Gegenwartsliteratur und sie geben der österreichischen Literatur literarisch und ästhetisch interessante Impulse. Viele von ihnen wollen sich unter neuen kulturellen Bedingungen, in einer anderen Umwelt durchsetzen, sich im Kulturkreis der neuen Heimat positionieren oder vielleicht sogar ihre kulturelle Identität neu entwerfen. In ihren Texten kann man neben Thematisierungen eines Zustands der kulturellen Verpflanzung und Neuverortung auch der Auseinandersetzung mit Fragen der nationalen und kulturellen Identität finden. In dem Beitrag wird der Frage nachgegangen, mit welchen Identitätsentwürfen der Leser in den Texten von einem inzwischen im deutschsprachigen Literaturbetrieb etablierten Autor mit Migrationshintergrund konfrontiert wird: Gegenstand der Analyse bilden ausgewählte Texte von Radek Knapp, einem literarischen „Brückenbauer“ des polnisch-österreichischen Kulturraums. Die ausgewählten literarischen Texte Knapps (sein erster - Herrn Kukas Empfehlungen - sowie sein bisher letzter Roman - Der Gipfeldieb) werden daraufhin befragt, wie (und ob) die dargestellten Figuren ihre Identität herstellen oder bewahren sowie wie Radek Knapp auf das „Österreichische“ ein- bzw. mit dem „Österreichischen“ umgeht.
\end{abstract}

SCHLÜSSELWÖRTER: österreichisch-polnische Literaturbeziehungen, österreichische Gegenwartsliteratur, kulturelle Identität, Migrantenliteratur, Schriftsteller mit dem Migrationshintergrund, Radek Knapp

\section{POLISH IMMIGRANTS IN AUSTRIAN LITERATURE: SOME REMARKS ABOUT AUS- TRIAN-POLISH LITERARY RELATIONSHIPS IN THE 21ST CENTURY}

ABSTRACT: Austria can look back on a long history of literature which is marked by its inter-/transcultural character. Many of the Austrian authors are cross-border commuters, their intercultural ties give them a "bridge-breaking" function: The literary texts of authors with a migration background

Agnieszka Palej - Uniwersytet Jagielloński, Kraków, agnieszka.palej@uj.edu.pl 
today form an integral part of Austrian contemporary literature and give literary and aesthetically interesting impulses to Austrian literature. Many of them want to assert themselves under new cultural conditions, in a different environment, to position themselves in the cultural circle of the new home, or perhaps even to redesign their cultural identity. In their texts one can find not only the themes of a state of cultural transplantation and relocation, but also the examination of questions of national and cultural identity. The article investigates which identity drafts the reader is confronted with in the texts by an author with a migration background who has meanwhile established themself in the German-language literary scene: The analysis focuses on selected texts by Radek Knapp, a literary "bridge builder" of the Polish-Austrian cultural area. The selected literary texts of Knapp are then analysed in order to see how (and if) the depicted figures establish or preserve their identity and how Radek Knapp approaches and deals with the "Austrian-ness".

KEYWORDS: Austrian-Polish literary relationships, Austrian contemporary literature, cultural identity, authors with a migration background, Radek Knapp

Österreich blickt auf eine lange Literaturgeschichte mit inter- / transkultureller Prägung zurück. „Bekanntlich ist die österreichische Kultur von Inhalten bestimmt, die aus den Subkulturen der verschiedenen Ethnien der mitteleuropäischen Region stammen. Es sind nicht nur deutsche oder alpenländische, sondern auch ungarische, böhmische, polnische, kroatische, italienische und selbstverständlich auch jüdische kulturelle Codes in ihr enthalten, die sich zu einer neuen kulturellen Konfiguration zusammenfügen“ - so Moritz Csáky (Csáky 1996: 115). Der Vielvölkerstaat Österreich bildete in der europäischen Kulturgeschichte im 18. und 19. Jahrhundert einen Raum der intensiven kulturellen Kommunikation. Die Phänomene der Transkulturalität, Migration, Pluriethnizität, Multilingualität sind im Laufe der Zeit zu Komponenten des kollektiven Bewusstseins geworden. Die einzelnen Völker, die auf dem Gebiet der Monarchie gelebt haben, tragen heute noch das Erbe dieses spezifischen kulturellen Kommunikationsraumes in sich. Dieses historisch-kulturelle Erbe wirkte sich auf die österreichische Identität aus. Die identitätsbildende Rolle wird vor allem auf die Habsburger Monarchie und deren Staatsapparat zurückgeführt. ${ }^{1}$ Das Bestehen der österreichisch-ungarischen Monarchie stellte eine materielle Basis für die Entwicklung einer mitteleuropäischen Identität dar, die auch die polnische kulturelle Identität beeinflusst. Die österreichisch-polnischen kulturellen Beziehungen sind nämlich von einer übergreifenden Nähe gekennzeichnet: Österreich und Polen sind historisch viele Jahrhunderte eng miteinander verbunden gewesen. Zur Konfrontation und Begegnung zweier Kulturen kam es insbesondere infolge der ersten (1772) und dann der 3. Teilung Polens. Seit der Zeit der Teilungen Polens und der österreichischen Eingliederung "Galiziens und Lodomeriens" gewann die Bedeutung des Wiener Bildes im Nationalbewusstsein sowie in der polnischen kulturellen Tradition an Intensität. Wien war das

1 „Das kollektive Gedächtnis der Österreicher speiste sich und speist sich immer noch aus historischen Erfahrungen, Diskursen, Symbolen und Klischees, die natürlich weit über die Perspektive des politischen und gesellschaftlichen Pluralismus hinausgehen.“ (Puchalski 2009: 10) 
Zentrum eines mitteleuropäischen Kulturraumes, das von gegenseitiger Durchdringung der verschiedenen Kulturen geprägt war. Die österreichische Hauptstadt mit ihrem Völkergemisch zog polnische Arbeiter, Intellektuelle und Künstler an. Manche Polen übten in Wien der Habsburger Monarchie höhere Staatsfunktionen aus, viele von ihnen bekleideten hohe Stellen in der österreichischen Exekutive, Legislative und im juristischen System. ${ }^{2}$ Die gemeinsamen (polnisch-österreichischen) historischen existentiellen Grunderfahrungen spielen hier eine wichtige Rolle, weil sie im Laufe der Zeit von Kunst und Literatur zu erkennbaren Kulturzeichen aufgearbeitet und zu Komponenten des kollektiven Bewusstseins wurden. ${ }^{3}$ In Folge dieses intensiven Kulturtransfers sowie der kulturübergreifenden Kommunikation sind ,gemeinsame” Räume von ethnisch-kultureller Vielfalt (ehem. Galizien) als kulturell multiple Symbiosen sowie multiple kulturelle Identitäten entstanden und es haben sich nach und nach Konstrukte herausgebildet, die Identität als pluriell und in ständigem Wandel begriffen verstehen. Die transkulturellen Verbindungen manifestierten sich auch in den grenzüberschreitenden Biografien. Die prominentesten Bespiele des Transkulturellen im literarischen Bereich sind wahrscheinlich Stanisław Przybyszewski (1868-1927), Tadeusz Rittner (1873-1921), die ihre literarischen Texte auf Deutsch und auf Polnisch verfassten und deren Biografien und Werke eng mit dem deutsch-polnischen (Przybyszewski) und polnisch-österreichischen (Rittner) Kulturraum verbunden sind. Nach dem Zweiten Weltkrieg kam es zu einschneidenden Grenzveränderungen und Bevölkerungstransfer und die gesellschaftspolitischen, wirtschaftlichen, konfessionellen und nationalen Verhältnisse in Ostmitteleuropa veränderten sich grundsätzlich. Im Gegensatz zu den deutsch-polnischen belastete der Zweite Weltkrieg die polnisch-österreichischen Nachkriegsbeziehungen weniger. Die Besetzung Österreichs - ebenso wie Deutschlands - 1945 durch die Armeen der Siegermächte, die Aufteilung in Besatzungszonen und endlich die Zusicherung der ,immerwährenden Neutralität“ des österreichischen Staates im österreichischen Staatsvertrag (1955) eröffneten ein neues Kapitel der österreichisch-polnischen Beziehungen. Trotz der gesellschaftspolitischen Zuordnung in Europa nach 1945 sind die politischen und kulturellen Beziehungen zwischen Polen und Österreich im Vergleich mit denen zwischen Polen und der Bundesrepublik

\footnotetext{
${ }^{2} \mathrm{Zu}$ den prominentesten Persönlichkeiten, die politische Karrieren in Wien machten und in höchste k.u.k. Ämter gelangten, gehören u. a.: Karol Lanckoroński - Vizepräsident des Staatsdenkmalamtes und Generalkonservator für Galizien, Franciszek Smolka - Präsident des k.u.k. Parlaments (1848-1849), Agenor Gołuchowski (der Ältere) - österreichischer Minister für Inneres (1859-1860) und Statthalter von Galizien (1849-1859, 1866-1868 und 1871-1875) Agenor Gołuchowski (der Jüngere) - k.u.k. gemeinsamer Außenminister Österreich-Ungarns (1895-1906), Alfred Józef Potocki - Premierminister (1870-1871), Kazimierz Badeni - Premierminister (1895-1897), Julian Dunajewski - Finanzminister (1880-1891), Leon Biliński österreichischer Finanzminister (1895-1899 im Kabinett Badeni und 1909-1911 im Kabinett Bienerth), k.u.k. gemeinsamer Finanzminister Österreich-Ungarns (1912-1915).

${ }^{3}$ An dieser Stelle muss auf die schwärmerische, nostalgische Verklärung einstiger Plurikulturalität der Habsburger Monarchie aufmerksam gemacht werden, wie sie im polnischen Kollektivbewusstsein nach dem Zweiten Weltkrieg besonders zur Wirkung kam.
} 
Deutschland weniger problematisch gewesen. Dabei hat - trotz der Westorientierung der Republik Österreich und der Zeit des Bestehens des sog. Eisernen Vorhanges - die österreichische Verpflichtung zu einer ,immerwährenden Neutralität" eine wesentliche Rolle gespielt. Auf Grund seines Neutralitätsstatus konnten im Rahmen der österreichisch-polnischen Beziehungen mit den Staaten des Warschauer Pakts Kooperationsbereiche entwickelt werden, weil Österreich sich unter den sog. westlichen Staaten einer Sonderstellung erfreute. Viele polnische Intellektuelle (u. a. Aleksander Jackiewicz, Stanisław Jerzy Lec, der in den vierziger Jahren als polnischer Presse- und Kulturattaché in Wien arbeitete, und Stanisław Lem, der sich in Österreich als Schriftsteller großer Popularität erfreut) lieferten einen bedeutenden Beitrag zur Aufrechterhaltung des österreichisch-polnischen kulturellen Dialogs. Die in Österreich existente Demokratie westlichen Musters und der wirtschaftliche Erfolg stellten im Vergleich zu dem unter dem sowjetischen Einfluss stehenden polnischen Staat mit seinen Krisenjahren 1956, 1970, 1980/81, 1988/89 eine politische Alternative oder ökonomische Notwendigkeit für viele Polen dar, die nach Österreich ins Exil gingen. Wien war sozusagen ein historisch vertrautes Terrain, weil - wie schon oben erwähnt - ein Teil Polens bis 1918 staatspolitisch zum Habsburgerreich gehört hatte. Viele Einwanderer aus dem ostmitteleuropäischen Kulturkreis lebten in dem vor allem in Wien konzentrierten Völkergemisch und assimilierten sich somit an die österreichische Kultur. Polen war schon immer ein wichtiges Herkunftsland für Österreich, was auf die räumliche Nähe, die historischen Entwicklungen und - last but not least - auf die kulturellen Verbindungen zurückzuführen ist. Österreich entwickelte sich zu einem beliebten Einwanderungsland für die polnischen Migranten. Polen kamen als Flüchtlinge, Aussiedler, Vertriebene, Asylbewerber, Zwangs- oder Gastarbeiter. Darunter befanden sich auch zahlreiche Künstler, die z. B. das Gastland Österreich ${ }^{4}$ gewählt haben. Es stellt sich die Frage, ob die österreichisch-polnischen gegenseitigen kulturellen Berührungen, der seit dem Mittelalter bestehende Kulturaustausch sowie Vermischungen der Bewohner ihren Charakter seit dem Ende des Ost-West-Gegensatzes und im Zeitalter der Globalisierung, in dem nationalstaatliche Grenzen nach und nach ihre Relevanz verlieren, tatsächlich ihre Form radikal veränderten. Das österreichisch-polnische transnationale Aufeinandertreffen hat seine Wurzeln in den wechselseitigen Beziehungen in der Vergangenheit, die schon längst den transkulturellen Typus konstituiert hat.

In den letzten Jahren hat sich das Thema Migration (auch Migration in der Literatur) markant entwickelt. Vor allem seit den 1990er Jahren des 20. Jahrhunderts traten immer öfter immigrierte Autoren in die deutschsprachige Öffentlichkeit. Viele der sog. zugewanderten Schriftsteller fanden auch Eingang in die österreichische Literatur.

${ }^{4}$ In Österreich lebten für einen begrenzten Zeitraum oder dauerhaft u. a. folgende Schriftsteller (in jedem einzelnen Fall müssen die historischen Umstände in Betracht gezogen werden: die Existenz der Habsburger Monarchie (bis 1918) sowie (bis 1918) die Nichtexistenz eines unabhängigen polnischen Staates): Tadeusz Rittner (1873-1921), Ludwik Szczepański (1872-1954), Zenon Miriam Przesmycki (1861-1944), Adam Nowicki (1865-1949), Stanisław Lem (1921-2006), Adam Zieliński (1929-2010). 
Viele von ihnen stehen im Konflikt zwischen Vereinnahmung und Ausgrenzung. Sie sind aber Grenzgänger zwischen den Kulturen, ihre inter-bzw. transkulturelle Bindung verleiht ihnen eine „brückenschlagende“Funktion: Die literarischen Texte von Autoren mit Migrationshintergrund bilden heute einen festen Bestandteil der österreichischen Gegenwartsliteratur und sie geben der österreichischen Literatur literarisch und ästhetisch interessante Impulse. Man muss aber dabei betonen, dass es die österreichische Literatur als Monokultur auch in der Vergangenheit nie gegeben hat. Die Literatur in dem deutschsprachigen Raum - und in Österreich - wurde und wird von Immigranten verfasst und gelesen. Viele von ihnen kommen mit unterschiedlichen kulturellen Hintergründen und Erfahrungen und wollen sich unter neuen kulturellen Bedingungen, in einer anderen Umwelt durchsetzen, sich im Kulturkreis der neuen Heimat positionieren oder vielleicht sogar ihre kulturelle Identität neu entwerfen. Die kulturelle Identität ist dabei oft der Schlüssel, der zentrale Rolle im künstlerischen Schaffen der sog. Migrantenautoren spielt. Im thematischen Zentrum ihrer literarischen Texte stehen oft dabei die Frage nach der nationalen Identität, die Verarbeitung der eigenen Vergangenheit sowie die Auseinandersetzung zwischen der neuen und der eigenen Kultur. In den Texten kann man auch neben Thematisierungen eines Zustands der kulturellen Verpflanzung und Neuverortung auch der Auseinandersetzung mit Fragen der nationalen und kulturellen Identität finden. Den Diskussionen um die Ein-/Zuwanderung liegt auch die Frage nach dem „Österreichischen“ und seinen Grenzen zugrunde, die verschiebbar sind. Die Autoren mit dem sog. Migrationshintergrund erweitern nämlich die Auffassung des „Österreichischen“.

In dem vorliegenden Beitrag wird der Frage nachgegangen, mit welchen Identitätsentwürfen der Leser in ausgewählten Texten von einem inzwischen im deutschsprachigen (darunter auch dem österreichischen) Literaturbetrieb etablierten Autor mit Migrationshintergrund konfrontiert wird. Der Fokus richtet sich dabei auf einen der Schriftsteller mit dem sog. „MigrantInnenhintergrund“ der österreichischen Gegenwartsliteratur, nämlich Radek Knapp (und seine zwei ausgewählten literarischen Texte: seinen ersten - Herrn Kukas Empfehlungen und seinen bisher letzten Roman Der Gipfeldieb). Mich interessiert vor allem, wie (und ob) Knapps Protagonisten ihre (neue) Identität herstellen oder bewahren sowie wie der Schriftsteller Radek Knapp auf das „Österreichische“ ein- bzw. mit dem „Österreichischen“ umgeht und inwiefern das „Österreichische“ eine identitätsstiftende Funktion hat.

Radek Knapp, ein literarischer „Brückenbauer“ des polnisch-österreichischen Kulturraums und Chamisso-Preisträger (2000) ${ }^{5}$, wurde 1964 in Warschau geboren und lebt heute in Wien. Er hat in seiner Biographie einen Sprachwechsel vollzogen und als Literatursprache bewusst die Sprache seiner neuen österreichischen Heimat gewählt. Der Schriftsteller betrachtet das Deutsche als seine Arbeitssprache (Knapp

${ }^{5}$ Der Adelbert-von-Chamisso-Preis wird seit 1985 den deutschschreibenden Autoren nichtdeutscher Herkunft und Muttersprache für ihre herausragenden literarischen Leistungen zuerkannt. 
1996: 147). Knapp schreibt seine Texte oft von seinem kulturellen (als polnisch markierten) Hintergrund aus. Das eigenkulturelle Element wird auch in den Interviews besonders hervorgehoben und zu einer bewussten Selbststilisierung erhoben. Das Leben in verschiedenen Sprachen und Kulturen ermöglicht den Migrantenautoren, in die Rolle eines Vermittlers zwischen Kulturen und Sprachen zu schlüpfen. Der Schriftsteller Radek Knapp weigert sich nicht, auf seine Herkunft hinzuweisen. Er betont sogar sein Polnischsein und übernimmt anscheinend gern die Funktion eines literarischen Kulturvermittlers und pendelt - wie er es selbst nennt - zwischen zwei Kulturen: „der unverbrauchten und etwas chaotischen slawischen und der westlichen, die sehr geordnet, aber manchmal auch ein bisschen langweilig ist." (Wörgötter 1997: 8) Der Schriftsteller Knapp macht seine polnische Herkunft zum Markenzeichen seiner schriftstellerischen Tätigkeit, um sich wahrscheinlich auf diese Weise innerhalb des deutschsprachigen Literaturbetriebs zu positionieren. In seinem Falle kann man den Eindruck gewinnen, dass er weniger die Vermittlung künstlerisch-ästhetischer Werte in das Zentrum seines literarischen Wirkens stellt, sondern vielmehr seine Aufgabe eben darin sieht, sein neugewonnenes österreichisches Heimatland für die Belange und Erfahrungen Polens bzw. Ostmitteleuropas zu sensibilisieren.

Wie schon erwähnt, hat Radek Knapp als Kind seine alte Heimat verlassen und in Österreich einen neuen Lebensabschnitt angefangen. Dementsprechend sind seine literarischen Figuren Grenzgänger und Zuwanderer in einem neuen Land/in Österreich und seine Texte behandeln Bewegungen zwischen zwei Kulturkreisen. ${ }^{6}$ Knapps Protagonisten sind Betroffene, die sich zwischen Ost und West, zwischen zwei Kulturen bewegen, die kulturelle Fremde und die neuen Kulturcodes „zähmen“ lernen müssen und in den meisten Fällen über ihr Befinden in dem neuen - österreichischen - Kulturraum, in der Fremde, berichten. Seine literarischen Texte kreisen dabei um polnische Stereotype in Österreich, Kontraste zwischen Polen und Österreich, bzw. der sog. Östlichen/slawischen und westlichen Welt, Klischeebildung usw. Der Schriftsteller entwirft dabei auf ironisch-satirische Weise ein Bild von Österreich und den Österreichern, insbesondere von Wien und seinen Bewohnern. Die Klischees sind Teil einer wirklichen - wenn auch übertriebenen - Erfahrung und Knapps ironisches Spiel mit ihnen seine literarische Reaktion auf bestimmte festgefahrene Vorstellungen. Es ist ein Blick von außen und innen zugleich, der Blick eines seit seinem zwölften Lebensjahr in Wien lebenden Zuwanderers, der aus der nötigen Distanz seine Ironie gelten lässt. Knapp spielt bewusst mit den Stereotypen, das klischeehafte Gesamtbild basiert aber auf positiven und weithin bekannten Assoziationen. Es muss dabei betont werden, dass

${ }^{6}$ Der Schriftsteller hat bereits vier Romane (Herrn Kukas Empfehlungen 1999, Der Papiertiger 2003, Reise nach Kalino 2012, Der Gipfeldieb 2015), einen Erzählungsband (Franio 1994), einen essayistischen Reiseführer auf Deutsch geschrieben (Gebrauchsanweisung für Polen 2005) sowie Erzählungen Der Mann, der Luft zum Frühstück aß. Erzählung 2017, und Die Stunde der Geburt/The Hour Of Birth. Eine Erzählung zu 41 Grafiken von Alfred Kubin (2017) veröffentlicht. Sein Roman Herrn Kukas Empfehlungen gehört zu den erfolgreichsten Longsellern bei Piper-Verlag. 
der Gebrauch von klischeehaften Elementen zu den hervorstechenden Merkmalen der literarischen Werkstatt des Schriftstellers gehört, die er bewusst und konsequent in seine Texte einbaut und daraus literarische Figuren, ihre Charaktere und Handlungsstränge konstruiert. Der Schriftsteller erzielt seine Effekte bewusst, indem er Naivität der Darstellung simuliert. Er simuliert die Naivität der Darstellung, bauscht die Klischees so auf, dass die darin steckende Absurdität klar wird. Knapps Literatur reflektiert in besonderer Weise die Situation als Zuwanderer und Integrierte. Der Großteil seiner literarischen Texte kreist um die Situation von einem Migranten/Einwanderer in seiner österreichischen Heimat, besonders um solche, die in Polen geboren sind und als Kinder bzw. junge Menschen nach Österreich kommen, sich in der Fremde behaupten müssen und infolgedessen Teil der neuen Heimat werden, d. h. hier ihre neue Identität finden. Vergleicht man die Biographie des Autors mit dem Lebensweg seiner Ich-Figuren, legen seine literarischen Texte - wie schon erwähnt - grundlegende autobiographische Züge nahe. Die Handlungen seiner literarischen Texte spielen in der Donaumetropole, der Wahlheimat des Autors. Knapps Protagonisten kommen oder leben bereits in Wien und erleben die Donaumetropole ab einem bestimmten Zeitpunkt ihrer Biographie als ihren Lebensmittelpunkt und sind damit einem Prozess der kulturellen Anpassung ausgesetzt und finden früher oder später Anschluss an ihre österreichische Heimat. Die Gegenüberstellung der beiden (polnischen sowie österreichischen) Welten ist auch in vielen seinen Texten, in denen er sich mit seinem Herkunftsland Polen sowie mit seiner neuen Heimat, Österreich bzw. Westeuropa, literarisch auseinandersetzt, das zentrale Thema, wobei sich seine ironische Distanz zu den beiden von ihm beobachteten und erfahrenen kulturellen Wirklichkeiten doch bemerken lässt. Knapps Prosawerke sind eine ironisch-kritische Reflexion über die eigentümlichen sozial- und kulturspezifischen Differenzen der beiden kontrastierten Gesellschaften, Polens und Österreichs, oder besser des sog. Westens und Ostens. Knapps Figuren bewältigen den Weltenwechsel zwischen Ost und West. Die Relation zwischen der neuen und der alten Heimat sowie die Suche nach der neuen Identität werden selbst zum Thema.

Waldemar/Waldi, der Ich-Erzähler des ersten Romans Herrn Kukas Empfehlungen (1999), für den der Schriftsteller 2001 mit dem Adelbert-von-Chamisso-Förderpreis ausgezeichnet wurde, verlässt das vertraute eigene nationale und kulturelle Universum Polens und fährt in den Westen, nach Wien, um auf diesem Wege die eigene Identität zu finden. Die Wiener Lebensart, die traditionsreiche Stadt und deren Lebenswelt, das ersehnte Land des Wohlstands wirken sich auf den Protagonisten aus, die er in einer für Knapps Figuren typischen Art und Weise beschreibt: voller Begeisterung und unschuldiger Naivität. Der Protagonist/Waldemar verlässt seine Ursprungskultur, versucht aber seinen eigenen Weg in einem fremden Kulturkreis zu finden, bewältigt die Aneignung des Fremden und lernt allmählich die neue Kultur kennen. Die Spannung zwischen dem Eigenen und dem Fremden ist aber nicht leicht auszuhalten und ruft auch existentielle Angst vor dem Scheitern hervor. Der junge Mann, der sich auf dem Weg zu menschlicher und zugleich kultureller Vollwertigkeit befindet, muss sich in der für ihn 
anfangs feindlichen und bedrohlich erscheinenden Umwelt bewähren, um am Ende dieser Übergangsphase ein vollkommenes Individuum zu werden, das mit wichtigen Normen und Werten der Gesellschaft vertraut ist. Der naive, unerfahrene Waldemar muss (als Ausländer in Wien) ums Überleben in der westlichen Welt kämpfen, muss sich bewähren und versucht sein Glück in allen möglichen Unternehmungen. In der österreichischen Fremde wird er zum Außenseiter und ist gezwungen, mit illegalen oder halblegalen Mitteln zu überleben. Er tut dies ohne besonderen Plan, sondern von Fall zu Fall, von Not gedrungen. Die kulturelle Zugehörigkeit der Protagonisten scheint am Anfang eine Rolle zu spielen, kulturelle Differenzen zwischen dem kulturell „Eigenen“ und dem „Fremden“ werden markiert. Waldemar ist als Fremder äußerlich ebenfalls sozusagen „stigmatisiert“: Seine polnischen Turnschuhe bilden Stigmen in der westlichen Wiener Konsumwelt, die ihn in der „fremden“Welt gegenüber den Touristen besonders auffällig werden lassen: „Zwar besaß ich keine Sony-Kamera, keine Goretex-Jacke, nicht mal eine Sonnenbrille, aber dafür hatte ich dem österreichischen Fremdenverkehr zwei wachsame Augen und meine Tennisschuhe mit schwarzem Rand anzubieten.“ (Knapp 1999: 66)

Die „Ostblockschuhe“ (Knapp 1999: 90) werden als ein wichtiges Signal in der westlichen Welt betrachtet, das von der Umwelt sofort als etwas „Nicht-Eigenes“ entschlüsselt wird. ${ }^{7}$ Dieses Stück Kleidung symbolisiert den Status des vollmündigen Bürgers der westlichen Kultur, der am äußeren Erscheinungsbild abgelesen werden kann. Der Prozess der Aneignung des Fremden als Individuationsleistung vollzieht sich aber unaufhaltsam und der Protagonist beginnt neue Schuhe zu tragen. Im Verlauf des Selbstfindungsprozesses erweitert Waldemar seinen sozialen und kulturellen Erfahrungshorizont. Sinn und Bedeutung der Symbole des neuen Kulturraumes müssen erlernt und in ihrem Gebrauch erprobt werden. So erfährt Waldemar beispielweise, dass „Lipizzaner" kein „österreichisches Spezialdessert" seien, das man „unbedingt bestellen“ soll, wenn man in einem Kaffeehaus ist (Knapp 1999: 13). Das Wiener Kaffeehaus übrigens, das wichtigste Symbol der sog. Wiener Art, eine Institution, die untrennbar mit dem Stadtbild verbunden und eine Kulturform ist, an der ein Individuum, unabhängig von seiner ursprünglichen kulturellen Zugehörigkeit teilhaben kann, erfüllt im Prozess der Aufnahme in (eine) andere Kultur sowie bei der Erweiterung der kulturellen Identität eine wichtige Funktion. Der Besuch in einem Kaffeehaus ermöglicht dem Ich-Erzähler sogar eine Identifikation mit der ,anderen“ Kultur. Das Wiener Kaffeehaus vermittelt ein „Glücksgefühl“ (Knapp 1999: 81). Waldemar kann ,endlich wie ein Wiener an einer Melange nippen“ (Knapp 1999: 72), atmet „die gleiche Luft wie die reichen Westler“ (Knapp 1999: 81). Dank dem Kaffeehaus-Besuch überwindet er sein „Fremdsein“ und fühlt sich „schon ein bisschen wie ein echter Wiener“ (Knapp 1999: 82).

${ }^{7}$ „Wenn du es hier zu was bringen willst, musst du in deine Schuhe investieren“ (Knapp 1999: 114), erfährt der Hauptheld von einem erfahrenen Landsmann, dessen Schuhe schon „westlich“ aussehen: „Westqualität. Braunes Leder. [...] er hatte keine Jeans an, sondern eine alte Cordhose. Von jemandem wie ihm konnte man wirklich etwas lernen.“(Knapp 1999: 115) 
Der polnische Migrant Waldemar bemüht sich, seinen eigenen Lebensweg zu finden, entwickelt dabei eigene Sozialbeziehungen und wächst allmählich in die neue Kultur hinein. Er gewinnt neue Freunde außerhalb von Familie und Heimat, entdeckt in der kulturell neuen Welt die eigene Sexualität. ${ }^{8}$ Seine Liebeserlebnisse werden aber nicht nur auf das Sexuelle reduziert, der Migrant lernt in der kulturell neuen Welt die wahre Liebe kennen. Diese Wiener Liebe zu Irina, die übrigens große Ähnlichkeit zu der Herbstgrazie im Belvedere-Springbrunnen aufweist, kann eigentlich als ein entscheidender Faktor beim Eintritt des jungen Mannes in die Ordnung des neuen Kulturraumes und im Prozess der Akzeptanz der neuen Werte betrachtet werden.

Die symbolische Berührung des Marmorbauches dieser Skulptur des Springbrunnens im Belvedere-Park hat - schon am Anfang seines Wienaufenthaltes - die ,gute Laune“ des Protagonisten zur Folge (Knapp 1999: 81). Wegen ihrer verblüffenden Ähnlichkeit mit der Herbstgrazie aus dem Belvedere-Park hilft Irina als identitätsstiftendes Bindeglied zwischen der traditionsreichen kulturellen Vergangenheit und der Gegenwart der Aufnahmegesellschaft dem Protagonisten. Waldemar kann damit eine Synthese von Vergangenheit, Gegenwart und Zukunft finden und einen erheblichen Schub in seiner kulturellen Entwicklung erfahren. Der Aufenthalt in Wien hat die durch die Begegnung mit dem „kulturell Anderen“/dem „Österreichischen“ hervorgerufene Veränderung seiner Selbstidentität zur Folge. Die Schlussszene des Romans hat eine symbolische Bedeutung: Auf die Frage nach dem wahren Grund seiner Reise, dem Sinn seiner Grenzüberschreitung, antwortet Irina, die „Zwillingsschwester“ der „,dreihundert Jahre alte[n]“ Grazie aus Marmor (Knapp 1999: 246): „Um hier zu sein.“ (Knapp 1999: 251) Die österreichische Wiener Kulisse ermöglicht dem polnischen Migranten Waldemar, seine Identitätssuche erfolgreich zum Abschluss zu bringen.

In seinem bisher letzten Roman Der Gipfeldieb (2015), mit dem der Schriftsteller wieder zu seinen literarischen Anfängen zurückkehrt, erzählt Knapp die Geschichte eines Zuwanderers, seiner unfreiwilligen Emigration nach Wien sowie seiner Identitätsfindung. Der Ich-Erzähler, der 34-jährige gebürtige Pole Ludwik Wiewurka, lebt bereits seit seinem zwölften Lebensjahr in Wien. Im Gegensatz zu Waldemar aus Herrn Kukas Empfehlungen, der aus reiner Neugier nach Wien reiste, wurde Ludwik vor zweiundzwanzig Jahren von seiner Mutter der wohlbehüteten Obhut der polnischen Großeltern entzogen und nach Wien, ,,in ein fremdes Land“(Knapp 2015: 169),,entführt“ (Knapp 2015: 63). Der Romanprotagonist gerät in eine andere Welt mit anderer Sprache, anderen Mentalitäten, befindet sich ,plötzlich in einem fremden Land, unter fremden Leuten und muss innerhalb kürzester Zeit die Sprache des Feindes lernen“ (Knapp 2015: 63).

$\mathrm{Da}$ er als Kind alles zurücklassen musste, musste er sich immer neu definieren und ist auf der Suche nach seiner eigenen Identität sowie der Bedeutung des Wortes

\footnotetext{
${ }^{8}$ Waldemar erlebt ein flüchtiges sexuelles Abenteuer mit einer Landsmännin, die seine Mitbewohner als eine spezielle Überraschung zu seinem Geburtstag engagieren. Der bisher unerfahrene Jüngling wird von ihr verführt.
} 
„Zuhause“ (das er letztendlich aber doch in der österreichischen Fremde findet). Wie im Roman Herrn Kukas Empfehlungen wird auch hier die österreichische Hauptstadt zu demjenigen Ort kultureller Verschmelzung, in dem der Protagonist seine Identität in einem neuen Kontext wahrnehmen kann. Der Integrationsprozess von Ludwik Wiewurka verläuft allem Anschein nach ohne Probleme, er arbeitet als Heizungsableser und gewinnt dadurch den Zugang zu den Wiener Wohnungen und deren Inhabern, sogar zu ihren Seelen und zu den intimsten Lebensbereichen. Wiewurka kann aber in Wirklichkeit in Wien keine Wurzeln schlagen.

Wiewurka begegnet vielen Menschen und den ungewöhnlichsten Situationen, die ihn prägen und verändern, lernt über Grenzen, Geschmack und Gewohnheiten der Wiener Menschen. Doch hat er sich mit seinem Leben und Dasein in Wien eigentlich nie so recht abfinden können. Ludwik geht davon aus, dass zwar seine Emigration ,verjährt“ (Knapp 2015: 18) sei, weil er in der „Plüschstadt“ Wien (Knapp 2015: 6), „ein[em] groß[en] Museum“ (Knapp 2015: 26), „viel zu lange“ sei, um sich „noch als Emigrant zu fühlen“ (Knapp 2015: 18), obwohl er ,,seit zwanzig Jahren vergeblich“" versucht, „[s]einen Akzent aus[zu]löschen“ (Knapp 2015: 19). Wiewurka selbst kommt sich ,wie eine trübe Suppe [vor], die ein paar betrunkene Köche gekocht haben“" (Knapp 2015: 194). Wie sich zeigt, ist er selbst ein in sich zerrissener Mensch, bei dem ein (Militär)psychologe ,den Konflikt zwischen alter und neuer Heimat“ (Knapp 2015: 67) diagnostiziert. Nach fünfzehn Jahren Wartezeit wird ihm endlich die österreichische Staatsbürgerschaft verliehen. In einem ,geräumige[n] Büro mit imposanten alten Möbeln“, in dessen ,Zimmerdecke [...] der österreichische Adler von der Größe einer Cessna eingearbeitet" ist (Knapp 2015: 29f.), gelobt Wiewurka, seinem „Land Österreich zu dienen, in Friedenszeiten und im Krieg, [seine] Pflichten als Bürger wahrzunehmen und die Rechte zu schützen.“ (Knapp 2015: 32) Dies ist vor allem für seine Mutter von besonderer Bedeutung, die will, dass ihr Sohn endlich Österreicher wird und sich endlich gut in seiner Haut fühlt. Die unerwartete und im Grunde genommen aufgezwungene österreichische Staatsbürgerschaft stellt aber keine besondere Integrationsleistung dar und hilft dem Protagonisten bei seiner Identitätsfindung sowie seinem Selbstfindungsprozess nicht.

Erst sein Zivildienst in dem ,über hundert Jahre“ (Knapp 2015: 104) alten Wiener Altershaus „Weiße Tulpe“ im 19. Wiener Bezirk, dem „älteste[n] Altersheim im deutschsprachigen Raum“ (Knapp 2015: 104), in dem an der Wand ein großes Gemälde mit einem „Mann mit mächtigen Koteletten“ (Knapp 2015: 102) hängt, ermöglicht dem Romanprotagonisten eine Identifikation mit der österreichischen Heimat. Die österreichische Tradition wird zur Identitätsfindung des Protagonisten herangezogen, weil das traditionsreiche Wiener Altersheim den perfekten „Rahmen“ dafür bildet. Der frischgebackene österreichische Staatsbürger Ludwik Wiewurka, „ein Auto, das zu früh aus der Garage gefahren ist" (Knapp 2015: 169), findet in dem traditionsreichen Wiener Altersheim ,die perfekte Garage“ (Knapp 2015: 170). Es ist nämlich derjenige Ort, wo alles ,in Zeitlupe“ (Knapp 2015: 177) stattfindet und „eine angenehme Gravitation“ (Knapp 2015: 170) herrscht, „die von der Vergänglichkeit um einen herum 
erzeugt wird“ (Knapp 2015: 170). Die Figur findet in dem Altersheim vorübergehend „eine Zuflucht“ (Knapp 2015: 170) und „ein Zuhause“ (Knapp 2015: 193) und überwindet sein „Fremdsein“ sowie seine Angst vor der Zukunft. Wie im Falle von dem Protagonisten aus dem Roman Herrn Kukas Empfehlungen spielt auch in dem Roman Der Gipfeldieb bei der Identitätsfindung in der Fremde neben der Wiener Tradition auch eine Frau (und die Liebe zu ihr) eine wichtige Rolle. Es ist die aus Polen stammende Schwester Sylwia, wie Ludwik eine Emigrantin, die in dem Altersheim „Weiße Tulpe" als Krankenschwester arbeitet. Dem Protagonisten gelingt es somit, in der österreichischen Wiener Heimat seinen eigenen Weg zu finden und Ludwik entdeckt (wie Waldemar aus dem Debütroman von Knapp), dass seine Heimat überall (auch in Wien) sein kann. Das traditionsreiche Altersheim ist ein identitätsstiftendes Bindeglied zwischen der traditionsreichen kulturellen Vergangenheit und der Gegenwart der Aufnahmegesellschaft des Protagonisten. Wie Waldemar aus dem Roman Herrn Kukas Empfehlungen findet auch Wiewurka eine Synthese von Vergangenheit, Gegenwart und Zukunft. So kann die Arbeit in dem Altersheim als der entscheidende Faktor bei seinem Eintritt in die Ordnung des neuen Kulturraumes und im Prozess der Akzeptanz der neuen Werte betrachtet werden. Ein vor zweiundzwanzig Jahren von seinem Großvater gekaufter Fahrschein für seine Rückkehr aus Wien (Knapp 2015: 184), den der Protagonist in „einer Kiste mit Andenken aus [s]einer Kindheit“ (Knapp 2015: 181) aufbewahrt, wird in Anwesenheit seiner Mutter verbrannt. Seine Tat gewinnt einen identitätsstiftenden Symbolwert.

Beide Protagonisten Knapps - sowohl Ludwik wie auch Waldemar - verfügen über eine außerordentliche Fähigkeit, in sich verschiedene (polnische und österreichische), kulturelle Elemente zu vereinen und auf diese Weise eine neue Form der Identität aufzubauen. Eine entscheidende Rolle spielt die Wiener Kulisse. Wien, „ein großes Museum“ (Knapp 2015: 26), „ein stilles Wasser“ (Knapp 2015: 26), wird dabei nämlich zu demjenigen Ort, wo der polnische Migrant die eigene Identität findet. „Österreich ist häufig ein solcher Ort, wo man sich zu Hause fühlt, in jener Harmonie zwischen Vertrautheit und Ferne, wie sie Joseph Roth gefiel“ (Magris 1988: 204) - definiert Claudio Magris in dem Essayband Donau. Biographie eines Flusses den Begriff Österreich in Bezug auf die Heimfindung. So finden auch Knapps Protagonisten im „Österreichischen“ denjenigen Ort, wo man sich zu Hause fühlt, in jener Harmonie zwischen Vertrautheit und Ferne.

\section{Literatur}

Csáky, M. (1996). Ideologie der Operette und Wiener Moderne. Ein kulturhistorischer Essay zur österreichischen Identität. Wien, Köln, Weimar: Böhlau.

Knapp, R. (1996). ,, Wien und Warschau sind für mich zu einer Stadt zusammengeschmolzen “. Radek Knapp im Gespräch mit Christa Stippinger. In Ch. Stippinger (Hrsg.), Jeder ist anderswo ein Fremder. Interkulturelle Reihe des Vereins Exil im Amerlinghaus (S. 145-146). Wien: edition exil. 
Knapp, R. (1999). Herrn Kukas Empfehlungen. Roman. München: Piper.

Knapp, R. (2015). Der Gipfeldieb. Roman. München: Piper.

Magris, C. (1988). Donau. Biographie eines Flusses (H.-G. Held, Übers.). Wien: Zsolnay.

Palej, A. (2004). Interkulturelle Wechselbeziehungen zwischen Polen und Österreich im 20. Jahrhundert anhand der Werke von Thaddäus Rittner, Adam Zieliński und Radek Knapp. Wrocław: Oficyna Wydawnicza ATUT.

Puchalski, L. (2009). Vorwort. In L. Puchalski (Hrsg.), Ausgewählte Quellen im Diskursfeld »Identitäten«. Österreich. Ein Arbeitsbuch für Breslauer Germanisten (S. 9-10). Wrocław: Oficyna Wydawnicza ATUT .

Wörgötter, B. (27. November 1997). Vom Lachen, das in der Kehle stecken bleibt. Ein Pendler zwischen zwei Kulturen: Radek Knapp liest in Schwarz. Tiroler Tageszeitung, 8. 